\title{
Interferon- $\beta$ therapy and risk of thrombocytopenia in multiple sclerosis patients
}

\author{
Tatiana Koudriavtseva ${ }^{1}$ - Domenico Plantone ${ }^{1} \cdot$ Rosaria Renna $^{1} \cdot$ Chiara Mandoj $^{2} \cdot$ \\ Diana Giannarelli ${ }^{3}$ Caterina Mainero ${ }^{4,5}$
}

Received: 15 June 2015/ Accepted: 20 July 2015 / Published online: 26 July 2015

(C) Springer-Verlag Italia 2015

\begin{abstract}
Thrombocytopenia is a well-described adverse event of several disease-modifying therapies (DMT) in multiple sclerosis (MS). On the other hand, an increased prevalence of MS has been reported in patients with immune thrombocytopenia. In this retrospective, crosssectional, case-control study we evaluated in a heterogeneous MS cohort: (1) the prevalence of thrombocytopenia in comparison with sex- and age-matched controls; (2) the relationship between thrombocytopenia and patients' demographic, clinical characteristics; (3) the risk for thrombocytopenia in relation to DMT. 187 consecutive MS patients [51 males, mean age $( \pm \mathrm{SD}) 44.5 \pm 10.7$ years] and 200 controls (56 males, mean age $45.5 \pm 12$ years) were included. Thrombocytopenia was defined as platelet count lower than normal laboratory values $\left(130-400 \times 10^{9} / \mathrm{L}\right)$. The prevalence of thrombocytopenia was significantly higher in MS patients than in controls (7 vs. $2.5 \%, p=0.04)$. Thrombocytopenia was present only in relapsing-remitting MS cases, and significantly associated with lower $\operatorname{EDSS}(p=0.002)$ and with a trend for shorter disease duration $(p=0.06)$. It was more frequent in patients on high-dose interferon- $\beta$ therapy compared with
\end{abstract}

Tatiana Koudriavtseva

koudriavtseva@ifo.it

1 Multiple Sclerosis Center, Unit of Neurology, Regina Elena National Cancer Institute, IFO, Via Elio Chianesi 53, 00144 Rome, Italy

2 Clinical Pathology, Regina Elena National Cancer Institute, IFO, Rome, Italy

3 Scientific Direction, Regina Elena National Cancer Institute, IFO, Rome, Italy

4 Athinoula A. Martinos Center for Biomedical Imaging, Massachusetts General Hospital, Boston, MA, USA

5 Harvard Medical School, Boston, MA, USA those on low-dose interferon- $\beta$ therapy, other therapies or untreated patients $(p=0.02)$. High-dose interferon- $\beta$ therapy was associated with more than eightfold increase in the risk for thrombocytopenia (odds ratio 8.60, $95 \%$ confidence interval: 1.01-74.48 adjusted for EDSS, disease duration and type of disease). The prevalence of thrombocytopenia was increased in MS patients treated with DMT. High-dose interferon- $\beta$ therapy is the variable most strongly associated with thrombocytopenia.

Keywords Multiple sclerosis - Thrombocytopenia . Disease-modifying therapies $\cdot$ Beta-interferon

\section{Background}

Multiple sclerosis (MS) is an immune-mediated chronic disease of the central nervous system, pathologically characterized by inflammation, demyelination and neurodegeneration. Immune thrombotic thrombocytopenia (ITP) is a hematologic disorder characterized by isolated thrombocytopenia. It may be primary or may develop in association with other disorders, usually immune or infectious [1]. A previous study reported that MS prevalence in ITP patients is approximately 25 times higher than in the general population [2].

Thrombocytopenia has been also described as adverse event during several disease-modifying therapies (DMT) in MS such as interferon- $\beta$ (IFN- $\beta$ ) [3], cladribine [4], alemtuzumab [5], and fingolimod [6]. Conversely, drug-induced thrombocytopenia is uncommon in patients on teriflunomide [7], and has rarely been reported to be secondary to natalizumab [8,9] or glatiramer acetate treatment [10]. Furthermore, several case reports have been recently published on the occurrence of thrombotic microangiopathy with 
hemolytic-uremic syndrome and thrombotic thrombocytopenic purpura during IFN- $\beta$ therapy [11-18], leading to inclusion of this complication in the drug package leaflet by the EMA.

In this retrospective cross-sectional case-control study, based on the MS care and laboratory databases of Regina Elena National Cancer Institute in Rome, we aimed: (1) to compare the prevalence of thrombocytopenia in MS patients in comparison with age- and sex-matched controls; (2) to evaluate the relationship between thrombocytopenia and patients' demographic, clinical characteristics; (3) to evaluate the risk for thrombocytopenia in relation to different DMT, in particular to IFN- $\beta$ treatment, in order to verify in a clinical setting the data provided by trials. The study was conducted in accordance with the International Conference Harmonization Guidelines of Good Clinical Practice and the Declaration of Helsinki.

\section{Materials and methods}

\section{Participants}

187 consecutive MS patients who had a full blood count during the last outpatient assessment and 200 randomly selected age- and sex-matched controls, without a diagnosis of MS and having a full blood count, were included in the study. Patients affected by autoimmune diseases were excluded. Patients' neurological disability was evaluated by Expanded Disability Status Scale (EDSS).

To evaluate the risk of thrombocytopenia in relation to MS therapies, MS patients were characterized as the following: (a) no-therapy (untreated patients), (b) on therapy (all treated patients), (c) IFN- $\beta$ therapy (patients treated with Betaferon, Extavia, Rebif $44 \mu \mathrm{g}$, Avonex, Rebif $22 \mu \mathrm{g}$ ), (d) high-dose IFN- $\beta$ (patients treated with Betaferon, Extavia, Rebif $44 \mu \mathrm{g}$ ), (e) low-dose IFN- $\beta$ (patients treated with Avonex, Rebif $22 \mu \mathrm{g}$ ), (f) other therapies (patients treated with Copaxone, Tysabri, Gilenya).

\section{Laboratory data}

Thrombocytopenia was defined as a platelet count less than the lower limit of the laboratory normal range $\left(130-400 \times 10^{9} / \mathrm{L}\right)$. We defined "mild" thrombocytopenia as a platelet count between 100 and $130 \times 10^{9} / \mathrm{L}$, while "moderate" thrombocytopenia was defined as a platelet count between 99 and $50 \times 10^{9} / \mathrm{L}$ and "severe" $<50 \times 10^{9} / \mathrm{L}$.

\section{Statistical analysis}

Data were summarized using mean, standard deviation, median and range when referred to quantitative variables; categorical variables were summarized by means of absolute frequencies and percentages. The associations were analyzed by the Fisher's exact test or Chi-square test. Comparisons between groups were carried out for different variables using Student's $t$ test.

A multivariate logistic regression analysis was used to estimate drug-related risk of thrombocytopenia by diverse comparisons: (a) no-therapy vs. therapy; (b) no-therapy vs. IFN- $\beta$ therapy and other therapies; (c) no-therapy vs. highdose IFN- $\beta$, low-dose IFN- $\beta$ and other therapies; (d) notherapy vs. high-dose IFN- $\beta$ and other therapies + lowdose IFN- $\beta$. First, univariate analyses were performed for thrombocytopenia and demographic, clinical, and drug-related characteristics as well as for ongoing therapy and demographic, and clinical characteristics. Risk factors with significant relationships with both thrombocytopenia and therapy were used as adjustment factors for potential confounding in the logistic models. Odds ratios (OR) are presented with $95 \%$ confidence intervals (CI). An $\alpha$ level of 0.05 was considered to be statistically significant. All $p$ values are two-tailed. All statistical analyses were performed using SPSS version 20.0 (SPSS Inc., Chicago, IL, USA).

\section{Results}

187 consecutive MS patients [51 males, mean age $( \pm S D)$ $44.5 \pm 10.7$ years] and 200 sex- and age-matched controls (56 males, mean age $45.5 \pm 12$ years) were included in the study.

Patients' mean disease duration was $132.2 \pm 92.6$ months, mean EDSS was $3.1 \pm 2.2$ with a median (range) of $2.5(0-8)$.

There were 156 relapsing-remitting (RR), 27 secondaryprogressive (SP) and 4 primary-progressive (PP) MS patients. Fifty patients were treated with high-dose IFN- $\beta$, 32 with low-dose IFN- $\beta, 25$ with Copaxone, 22 with Tysabri and 13 with Gilenya; 45 patients were not on any DMT (untreated). Most untreated patients were SP and PP MS patients. The untreated RR MS patients refused any type of DMT. Twelve MS patients had a past history of cancer $(6.4 \%)$, but none of them had a cancer of hematological origin. No patient experienced a clinical relapse at the time of the full blood count and no patient was treated with high-dose of steroids during the prior 3 months.

We found positive correlations between age and disease duration $(r=0.48, p<0.0001)$, disease duration and EDSS $(r=0.36, p<0.0001)$, age and EDSS $(r=0.52$, $p<0.0001)$. Thrombocytopenia was not associated with anemia in MS patients (thrombocytopenia in $11.4 \%$ of anemic vs. $5.9 \%$ of non-anemic patients, $p=0.25$ ), as well as in controls (thrombocytopenia in none anemic vs. 
$2.8 \%$ of non-anemic subjects, $p=0.46)$. There were no significant associations between anemia and DMT $(p=0.68)$.

Treated patients had lower EDSS compared to the untreated ones $(2.9 \pm 2.1$ vs. $3.7 \pm 2.5, p=0.05)$, but they did not differ in age, gender, disease duration, and platelet count. Treated patients differed from untreated patients for disease type: the majority of RRMS (81.4\%) and SPMS $(55.6 \%)$ patients were treated, while all $(100 \%)$ PPMS patients were untreated $(p=0.001)$.

Thirteen of $187 \mathrm{MS}$ patients (7\%) and 5 of 200 controls $(2.5 \%)$ were thrombocytopenic, with the prevalence of thrombocytopenia significantly higher in patients ( $p=0.04)$. Thrombocytopenia was mild in 17 subjects and severe in one MS patient $\left(45 \times 10^{9} / \mathrm{L}\right)$, and was not associated with any clinically relevant event. None of MS patients with a past medical history of cancer had thrombocytopenia. Thrombocytopenia was found in $16 \%$ of patients treated with high-dose IFN- $\beta, 3.1 \%$ of those treated with low-dose IFN- $\beta, 9.1 \%$ of those treated with Tysabri, $4 \%$ of those treated with Copaxone, $2.2 \%$ of untreated patients and in none of Gilenya-treated patients.

Thrombocytopenia was found only in RRMS patients and was more frequent in patients on high-dose IFN- $\beta$ therapy compared with those on low-dose IFN- $\beta$ therapy, other therapies or untreated patients $(p=0.02$, Table 1$)$. It was also significantly associated with lower EDSS $(p=0.002)$, and with a trend for shorter disease duration $(p=0.06)$ (Table 1$)$. There were no significant associations between thrombocytopenia and type of disease, gender or age (Table 1).

At multivariate logistic regression analysis, high-dose IFN- $\beta$ therapy was associated with more than eightfold increase in the risk for thrombocytopenia (OR 8.38, $95 \%$ CI 1.01-69.92; and OR 8.60, $95 \%$ CI 1.01-74.48 adjusted for EDSS, disease duration and type of disease) when comparing untreated patients with those treated with highdose IFN- $\beta$, low-dose IFN- $\beta$ and other drugs as well as when comparing untreated patients with those treated with high-dose IFN- $\beta$ and other therapies + low-dose IFN- $\beta$ (OR 8.38, $95 \%$ CI 1.01-69.92, and OR 8.72, $95 \%$ CI 1.01-75.51 adjusted for EDSS, disease duration and type of disease) (Table 2).

\section{Discussion}

Thrombocytopenia is a well-described adverse event during several MS DMT and some of these treatments have been shown to modulate platelet or megakaryocytic functions [19-21].

Our study highlighted that high-dose IFN- $\beta$ therapy is the variable most strongly associated with thrombocytopenia, increasing more than eight times the risk for thrombocytopenia in our MS cohort. It is known that treatment with IFN- $\beta$ has been associated to asymptomatic reductions in almost all blood cell lineages and mild neutropenia, anemia, and thrombocytopenia were all reported to be statistically related to the treatment since the first drug trials on MS patients [3, 22]. Usually, thrombocytopenia is mild and generally resolves spontaneously while continuing therapy.

We found thrombocytopenia only in RRMS patients and an association with a lower disability score, with a trend towards significance for shorter disease duration. Indeed, a lower disability score is likely to be related to shorter disease duration, being EDSS and disease duration related factors. Moreover, both of them suggest a high probability of being treated with IFN- $\beta$. These results are in agreement with the known data that the inflammatory component is much more evident in RRMS than in the other disease forms and in the early phase that usually corresponds to lower EDSS. However, by adjusting for these factors at multivariate logistic regression analysis, high-dose IFN- $\beta$ therapy remained an associated risk for thrombocytopenia although the confidence interval was wide, likely reflecting that a bigger sample size would be needed. Furthermore, since the rate of thrombocytopenia in untreated patients $(2.2 \%)$ was similar to that in controls $(2.5 \%)$, it is reasonable to assume that its prevalence in our MS patients may depend prevalently on DMT and especially on highdose IFN- $\beta$ treatment. This confirms that IFN- $\beta$ may promote asymptomatic thrombocytopenia, which can reach up to the rare symptomatic cases of thrombotic thrombocytopenia reported in literature [11-18]. Despite thrombocytopenia has also been described during other DMT in MS, our study could not confirm these findings, probably due to the small number of patients in each treatment subgroup. Indeed, the population size is a limitation of this study.

On the other hand, platelet activation is known to be involved in the pathophysiology of MS, independently of treatment [23]. It is not clear whether this is an epiphenomenon, possibly secondary to endothelial injury in MS, or alternatively it is due to the role of platelets in neuroinflammation [24-26]. Platelets interact with leukocytes at the endothelium of the blood-brain barrier (BBB) and release the adhesion molecule PECAM-1 to the circulation is associated with leukocyte infiltration [24]. Moreover, platelet activating factor (PAF) can disrupt endothelial junctions and is likely to be one of the factors responsible for the early disruption of the BBB in MS. PAF can cause thrombocytopenia at levels as low as $3 \mathrm{ng} / \mathrm{kg}$, therefore it is not strange that mild thrombocytopenia reported for MS patients has been attributed to PAF activity [24]. Interestingly, PAF receptors are up-regulated in MS lesions [24]. 
Table 1 Relationships between platelet count and demographical and clinical characteristics of MS patients

\begin{tabular}{|c|c|c|c|}
\hline \multirow[t]{2}{*}{ Variable } & \multicolumn{2}{|l|}{ Platelet count } & \multirow[t]{2}{*}{$p$ value } \\
\hline & Normal $\left(130-400 \times 10^{9} / \mathrm{L}\right)(n=174)$ & Lower than normal $\left(<130 \times 10^{9} / \mathrm{L}\right)(n=13)$ & \\
\hline Age, years $($ mean $\pm S D)$ & $44.8 \pm 10.7$ & $40.6 \pm 9$ & $0.17 *$ \\
\hline Disease duration, months (mean $\pm \mathrm{SD}$ ) & $135.5 \pm 93.2$ & $86.7 \pm 77.5$ & $0.06^{*}$ \\
\hline EDSS (mean \pm SD) & $3.1 \pm 2.2$ & $2 \pm 1$ & $0.002 *$ \\
\hline Sex, $n(\%)$ & & & $0.35 * *$ \\
\hline Male & $46(90.2)$ & $5(9.8)$ & \\
\hline Female & $128(94.1)$ & $8(5.9)$ & \\
\hline Type of disease, $n(\%)$ & & & $0.25 * *$ \\
\hline Relapsing-remitting & $143(91.7)$ & $13(8.3)$ & \\
\hline Secondary progressive & $27(100)$ & $0(0)$ & \\
\hline Primary progressive & $4(100)$ & $0(0)$ & \\
\hline Therapy, $n(\%)$ & & & $0.02 * *$ \\
\hline High-dose IFN- $\beta$ & $42(84.0)$ & $8(16.0)$ & \\
\hline Low-dose IFN- $\beta$ & $31(96.9)$ & $1(3.1)$ & \\
\hline Other therapies & $57(95.0)$ & $3(5.0)$ & \\
\hline No-therapy & $44(97.8)$ & $1(2.2)$ & \\
\hline
\end{tabular}

EDSS Expanded Disability Status Scale

* Student's $t$ test

** Fisher exact test or Chi-square test

Table 2 Drug-related risk of thrombocytopenia in multiple sclerosis patients

\begin{tabular}{lll}
\hline Variable & Unadjusted OR $(95 \% \mathrm{CI})$ & $\begin{array}{l}\text { OR adjusted for EDSS, disease } \\
\text { duration and type of disease }(95 \% \text { CI })\end{array}$ \\
\hline $\begin{array}{l}\text { Therapy } \\
\text { No-therapy }\end{array}$ & 1 & 1 \\
Therapy & $4.06(0.51-32.14)$ & $3.73(0.46-30.04)$ \\
Therapy & 1 & 1 \\
No-therapy & $2.32(0.23-23.03)$ & $2.26(0.22-22.73)$ \\
Other therapies & $5.42(0.66-44.28)$ & $5.02(0.59-42.62)$ \\
High-dose + low-dose IFN- $\beta$ & 1 & 1 \\
Therapy & $1.32(0.23-23.03)$ & $2.29(0.23-23.12)$ \\
No-therapy & $1.42(0.08-23.57)$ & $1.03(0.06-17.96)$ \\
Other therapies & $8.38(1.01-69.92)$ & $8.60(1.01-74.48)$ \\
Low-dose IFN- $\beta$ & & 1 \\
High-dose IFN- $\beta$ & 1 & $1.78(0.19-16.71)$ \\
Therapy & $2.0(0.22-18.41)$ & $8.72(1.01-75.51)$ \\
No-therapy & $8.38(1.01-69.92)$ & 0.05 \\
Other therapies + low-dose IFN- $\beta$ & & \\
High-dose IFN- $\beta$ & &
\end{tabular}

No-therapy: untreated patients, therapy: all treated patients, high-dose IFN- $\beta$ : patients treated with Betaferon, Extavia, Rebif $44 \mu \mathrm{g}$, low-dose IFN- $\beta$ : patients treated with Avonex, Rebif $22 \mu \mathrm{g}$, other therapies: patients treated with Copaxone, Tysabri, Gilenya

$O R$ odds ratio, $C I$ confidence interval

* Multiple logistic regression analyses

A possible explanation for the association of thrombocytopenia with IFN- $\beta$ therapy could be the increased consumption of platelets due to activation of inflammatory- thrombotic processes since the physiological production of type I interferons and inflammatory cytokines starts the innate immune response [27]. Innate immunity represents 
the non-specific defense against infections and tissue damage acting through its essential processes including inflammation and blood coagulation [28]. Platelets are among the main effector cells not only in coagulation, but also in inflammation and in the continuum between innate and adaptive immunity [29]. Therefore, it is reasonable to hypothesize that as far both IFN- $\beta$ and pathophysiological MS processes contribute to induce thrombocytopenia in MS patients, the drug effect is prevalent through the increased activation of innate immunity.

In fact, in cases with high clinical or MRI inflammatory activity we usually prescribed different DMT rather than IFN- $\beta$, such as Tysabri or Gilenya, but they have been found to be related to a lower (Tysabri) or no risk (Gilenya) of thrombocytopenia. Therefore, the disease inflammatory component cannot be considered a main determinant of thrombocytopenia. Interestingly, we found thrombocytopenia in the $9.1 \%$ of patients treated with Tysabri. In the literature there are two reports describing drug-induced immune thrombocytopenia during treatment with Tysabri $[8,9]$. In both cases there was a good response to steroid treatment with rapid improvement of thrombocyte levels. Even if in our patients it was a mild asymptomatic thrombocytopenia, our data suggest that asymptomatic thrombocytopenia during treatment with natalizumab may be underestimated.

The present study highlights the importance of a close monitoring of the platelet count, especially during highdose IFN- $\beta$ therapy, to prevent the symptomatic thrombotic cases, strengthening the EMA recommendations on thrombocytopenia control in MS patients. However, further studies are needed in order to better identify the population at risk and understand the pathophysiological mechanisms of the thrombocytopenia in MS.

\section{Compliance with ethical standards}

Conflict of interest No conflict of interest or financial interest is reported. T.K. reports consulting fees from Bayer Schering, and Institutional grant from Merck Serono, Biogen Idec, Novartis, Bayer Schering outside the submitted work. C.M. reports consulting fees from Biogen outside the submitted work. Other authors have nothing to declare.

\section{References}

1. Kistangari G, McCrae KR (2013) Immune thrombocytopenia. Hematol Oncol Clin North Am 27(3):495-520. doi:10.1016/j.hoc. 2013.03.001

2. Segal JB, Powe NR (2006) Prevalence of immune thrombocytopenia: analyses of administrative data. J Thromb Haemost 4(11):2377-2383

3. Rieckmann P, O'Connor P, Francis GS, Wetherill G, Alteri E (2004) Haematological effects of interferon-beta-1a (Rebif) therapy in multiple sclerosis. Drug Saf 27(10):745-756
4. Langtry HD, Lamb HM (1998) Cladribine: a review of its use in multiple sclerosis. BioDrugs 9(5):419-433

5. Coles AJ (2013) Alemtuzumab treatment of multiple sclerosis. Semin Neurol 33(1):66-73. doi:10.1055/s-0033-1343797

6. European Medicines Agency: EMA/405985/2014 - Gilenya Scientific conclusions and grounds recommending the variation to the terms of the marketing. http://www.ema.europa.eu/docs/en GB/document_library/EPAR_-_Scientific_Conclusion/human/ 002202/WC500169779.pdf

7. European Medicines Agency: EMA/529295/2013 - Assessment report AUBAGIO International nonproprietaryname: TERIFLUNOMIDE. http://www.ema.europa.eu/docs/en_GB/docu ment_library/EPAR_-_Public_assessment_report/human/002514/ WC500148684.pdf

8. Cachia D, Izzy S, Berriosmorales I, Ionete C (2014) Drug-induced thrombocytopenia secondary to natalizumab treatment. BMJ Case Rep. doi:10.1136/bcr-2013-203313

9. Stosic M, De Jesus P, McCarthy J, Hutton G, Rivera V (2011) Immune thrombocytopenic purpura in a patient with multiple sclerosis treated with natalizumab. Neurology 77(5):505-507. doi:10.1212/WNL.0b013e318227b23f

10. Nguyen V, Bailey R, Sprague C (2013) P01 multiple sclerosis: treatment safety glatiramer acetate induced thrombocytopenia (P01.187). 80(Meeting Abstracts 1):P01.187

11. Sahraian MA, Eshaghi A (2010) Concomitant multiple sclerosis and idiopathic thrombocytopenic purpura. Eur $\mathrm{J}$ Neurol 17(8):e62-e63. doi:10.1111/j.1468-1331.2010.03098.x

12. Broughton A, Cosyns JP, Jadoul M (2011) Thrombotic microangiopathy induced by long-term interferon- $\beta$ therapy for multiple sclerosis: a case report. Clin Nephrol 76(5):396-400

13. Cavoli GL, Passantino R, Tortorici C et al (2012) Comment on thrombotic microangiopathy induced by interferon- $\beta$ therapy. Clin Nephrol 78(6):506-507

14. Nerrant E, Charif M, Ramay AS et al (2013) Hemolytic uremic syndrome: an unusual complication of interferon- $\beta$ treatment in a MS patient. J Neurol 260(7):1915-1916

15. Mahe J, Meurette A, Moreau A et al (2013) Renal thrombotic microangiopathy caused by interferon beta-1a treatment for multiple sclerosis. Drug Des Devel Ther 7(7):723-728

16. Larochelle C, Grand'maison F, Bernier GP et al (2014) Thrombotic thrombocytopenic purpura-hemolytic uremic syndrome in relapsing-remitting multiple sclerosis patients on high-dose interferon $\beta$. Mult Scler 20:1783-1787

17. Hunt D, Kavanagh D, Drummond I et al (2014) Thrombotic microangiopathy associated with interferon beta. N Engl J Med 370(13):1270-1271

18. Arrambide G (2014) Thrombotic thrombocytopenic purpurahaemolytic uremic syndrome in relapsing-remitting multiple sclerosis patients on high-dose interferon beta. Mult Scler 20(13):1788-1789. doi:10.1177/1352458514529614

19. Starossom SC, Veremeyko T, Dukhinova M, Yung AW, Ponomarev ED (2014) Glatiramer acetate (copaxone) modulates platelet activation and inhibits thrombin-induced calcium influx: possible role of copaxone in targeting platelets during autoimmune neuroinflammation. PLoS ONE 9(5):e96256. doi:10.1371/ journal.pone.0096256

20. Negrotto S, De Giusti CJ, Lapponi MJ, Etulain J, Rivadeneyra L, Pozner RG, Gomez RM, Schattner M (2011) Expression and functionality of type I interferon receptor in the megakaryocytic lineage. J Thromb Haemost 9(12):2477-2485. doi:10.1111/j. 1538-7836.2011.04530.x

21. Anada Y, Igarashi Y, Kihara A (2007) The immunomodulator FTY720 is phosphorylated and released from platelets. Eur J Pharmacol 568(1-3):106-111

22. The IFNB Multiple Sclerosis Study Group (1993) Interferon beta$1 \mathrm{~b}$ is effective in relapsing-remitting multiple sclerosis. I. Clinical 
results of a multicenter, randomized, double-blind, placebo-controlled trial. The IFNB Multiple Sclerosis Study Group. Neurology 43(4):655-661

23. Sheremata WA, Jy W, Horstman LL, Ahn YS, Alexander JS, Minagar A (2008) Evidence of platelet activation in multiple sclerosis. J Neuroinflamm 27(5):27. doi:10.1186/1742-2094-5-27

24. Horstman LL, Jy W, Ahn YS, Zivadinov R, Maghzi AH, Etemadifar M, Steven Alexander J, Minagar A (2010) Role of platelets in neuroinflammation: a wide-angle perspective. J Neuroinflamm 7:10. doi:10.1186/1742-2094-7-10

25. Nurden AT (2011) Platelets, inflammation and tissue regeneration. Thromb Haemost 105(Suppl 1):S13-S33. doi:10.1160/ THS10-11-0720
26. Behari M, Shrivastava M (2013) Role of platelets in neurodegenerative diseases: a universal pathophysiology. Int J Neurosci 123(5):287-299. doi:10.3109/00207454.2012.751534

27. O'Neill LA, Bowie AG (2010) Sensing and signaling in antiviral innate immunity. Curr Biol 20(7):R328-R333. doi:10.1016/j.cub. 2010.01.044

28. Esmon CT, Xu J, Lupu F (2011) Innate immunity and coagulation. J Thromb Haemost 9(Suppl 1):182-188. doi:10.1111/j.15387836.2011.04323.x

29. Vieira-de-Abreu A, Campbell RA, Weyrich AS, Zimmerman GA (2012) Platelets: versatile effector cells in hemostasis, inflammation, and the immune continuum. Semin Immunopathol. 34(1):5-30 